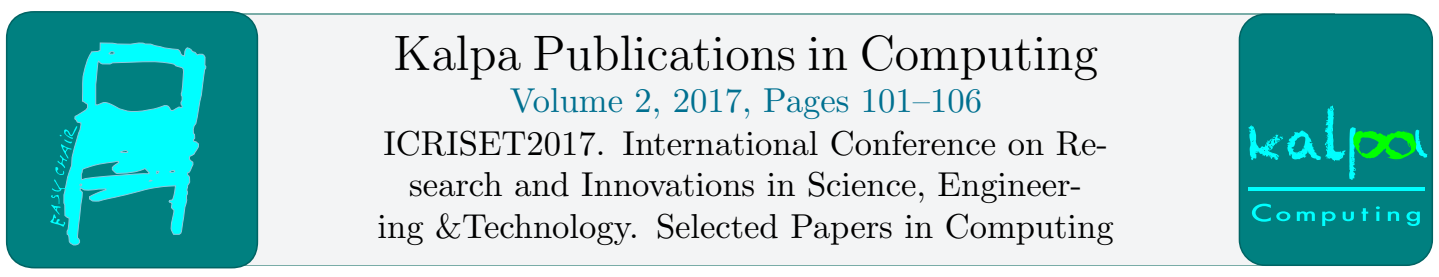

\title{
Study of radiation, reaction and parabolic motion effects on MHD Casson fluid flow with ramped wall temperature
}

\author{
Dr. Hari R. Kataria ${ }^{1}$ and Mr. Harshad R Patel ${ }^{2}$ \\ ${ }^{1,2}$ Department of mathematics, Faculty of science, The M. S. University of Baroda, Vadodara.
}

\begin{abstract}
This article studies effect of thermal radiation, chemical reaction and parabolic motion on the unsteady MHD Casson fluid flow past an infinite vertical plate embedded with ramped wall temperature. The fluid is electrically conducting and passing through a porous medium. This phenomenon modeled in the form of partial differential equations with initial and boundary conditions. Some suitable non-dimensional variables introduced and corresponding dimensionless equations solved using the Laplace transform technique. Analytical expressions for velocity, temperature and concentration profiles obtained. The features of the velocity, temperature and concentration are analyzed by plotting graphs and the physical aspects are studied for different parameters like the magnetic field parameter $\mathrm{M}$, thermal radiation parameter $\mathrm{R}$, chemical reaction parameter $R^{\prime}$, thermal Grashof number Gr, mass Grashof number Gm, Schmidt number Sc, Prandtl number Pr and time variable t.
\end{abstract}

\section{Introduction}

In nature, some non-Newtonian fluids behave like elastic solid that is, no flow occurs with small shear stress. Casson fluid is one of such fluids. This fluid has distinct features and is quite famous recently. Casson [1] introduced Casson fluid model for the prediction of the flow behavior of pigment-oil suspensions. Dash et al. [2] deals with Casson fluid flow in a pipe with a homogeneous porous medium.

The study of magneto-hydrodynamic flow of non-Newtonian fluid in a porous medium has attracted many researchers due to its application in the optimization of solidification processes of metals, alloys, the geothermal sources investigation and nuclear fuel debris treatment. Kataria and Patel [3] deal with MHD Second grade fluid flow through porous medium. Nadeem et al. [4] study with MHD flow of a Casson fluid in a porous medium. Das et al. [5] obtain analytic solution of chemical reaction and thermal radiation effects on MHD micropolar fluid in a rotating frame. Kataria and Mittal [6-7] discuss unsteady free convective MHD nano fluid flow whereas, Kataria et al. [8] consider magnetic field effects on micropolar fluid between two vertical walls. Muhaimin et al. [9] consider MHD Mixed convective flow with particle deposition and chemical reaction in a porous medium whereas, Muthucumaraswamy and Geetha [10] consider effects of parabolic motion on an 
isothermal vertical plate with constant mass flux. Recently, Kataria and Patel [11-12] deals with effect of radiation and chemical reaction on MHD Casson fluid flow with ramped wall temperature and ramped surface concentration through porous medium whereas, Kataria and Patel [13] discuss soret and parabolic motion effects on MHD Second grade fluid flow through porous medium.

Novelty of this paper is to study the important role of parabolic motion, radiation and chemical reaction on MHD flow of a Casson fluid flow with ramped wall temperature in a porous medium. We find the analytic solution using Laplace transform technique.

\section{Mathematical Formulation of the Problem}

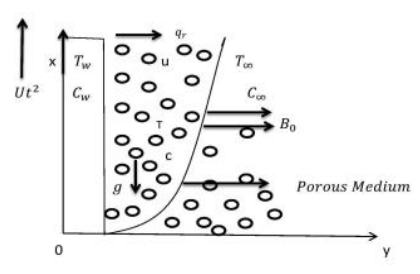

Figure 1: Physical Sketch
In Figure 1 the flow confined to $y^{\prime}>0$, where $y^{\prime}$ coordinate is measure in the normal direction to the plate and $x^{\prime}-$ axis taken along the wall in the upward direction. Initially, at time $t^{\prime}=0$, both the fluid and the plate are uniform temperature $T^{\prime}{ }_{\infty}$ and concentration $C_{\infty}^{\prime}$ at all the points respectively. A uniformly distributed transverse magnetic field of strength $B_{0}$ applied in the $y^{\prime}-$ axis direction.

At time $\mathrm{t}^{\prime}>0$, the plate is given an parabolic motion $\mathrm{U}_{0} t^{2}$ with heat transfer of the plate is raise or lowered to $\mathrm{T}_{\infty}{ }_{\infty}+\left(\mathrm{T}^{\prime}{ }_{\mathrm{w}}+\mathrm{T}^{\prime}{ }_{\infty}\right) \mathrm{t}^{\prime} / \mathrm{t}_{0}$ when $\mathrm{t}^{\prime} \leq \mathrm{t}_{0}$ and $\mathrm{T}^{\prime}{ }_{\mathrm{w}}$ when $\mathrm{t}^{\prime}>\mathrm{t}_{0}$ and level of the surface concentration at the plate linearly raise to $\mathrm{C}_{\infty}^{\prime}+\left(C^{\prime}{ }_{\mathrm{w}}+C^{\prime}{ }_{\infty}\right)$ which is there after maintained constant $\mathrm{T}^{\prime}{ }_{\mathrm{w}} \& C^{\prime}{ }_{\mathrm{w}}$ respectively. We assume that, rigid plate, incompressible flow, one dimensional flow, free convection and viscous dissipation term in the energy equation is neglected

Under above assumptions and taking into account the Boussinesq's approximation, governing partial differential equation with initial and boundary condition are

$\rho \frac{\partial u^{\prime}}{\partial t^{\prime}}=\mu_{B}\left(1+\frac{1}{\gamma}\right) \frac{\partial^{2} u^{\prime}}{\partial y^{2}}-\sigma B_{0}^{2} u^{\prime}-\frac{v \varphi}{k_{1}^{\prime}} u^{\prime}+\rho g \beta^{\prime}{ }_{T}\left(T^{\prime}-T^{\prime}{ }_{\infty}\right)+\rho g \beta_{C}^{\prime}\left(C^{\prime}-C^{\prime}{ }_{\infty}\right)$

$\frac{\partial T^{\prime}}{\partial t^{\prime}}=\frac{k}{\rho c_{p}} \frac{\partial^{2} T^{\prime}}{\partial y^{\prime 2}}-\frac{1}{\rho c_{p}} \frac{\partial q_{r}{ }^{\prime}}{\partial y^{\prime}}$

$\frac{\partial C^{\prime}}{\partial t^{\prime}}=D_{M} \frac{\partial^{2} C^{\prime}}{\partial y^{\prime 2}}-k_{2}^{\prime}\left(C^{\prime}-C_{\infty}^{\prime}\right)$

With following initial and boundary conditions:

$u^{\prime}=0, T^{\prime}=T_{\infty}^{\prime}, C^{\prime}=C_{\infty}^{\prime} ;$ as $y^{\prime} \geq 0$ and $t^{\prime} \leq 0, u^{\prime}=U_{0} t^{2}$, as $t^{\prime}>0$ and $y^{\prime}=0$, $C^{\prime}=C_{\infty}^{\prime}+\left(C^{\prime}{ }_{w}-C_{\infty}^{\prime}\right), T^{\prime}=\left\{\begin{array}{cc}T^{\prime}{ }_{\infty}+\left(T^{\prime}{ }_{w}-T^{\prime}{ }_{\infty}\right) t^{\prime} / t_{0} \text { if } 0<t^{\prime}<t_{0} ; y^{\prime}=0 \\ T^{\prime}{ }_{w} & \text { if } t^{\prime} \geq t_{0}\end{array} ; C^{\prime}\right.$ $u^{\prime} \rightarrow 0, T^{\prime} \rightarrow T_{\infty}^{\prime}, \quad C^{\prime} \rightarrow C_{\infty}^{\prime} ;$ as $y^{\prime} \rightarrow \infty$ and $t^{\prime} \geq 0$

The local radiant for the case of an optically thin gray gas expressed by Rosseland approximation [14] $\frac{\partial q_{r}{ }^{\prime}}{\partial y^{\prime}}=-4 a^{*} \sigma^{*}\left(T_{\infty}^{\prime 4}-T^{\prime 4}\right)$

Where $\sigma^{*}$ and $\mathrm{a}^{*}$ are Stefan Boltzmann constant and absorption coefficient respectively. Assuming that the temperature difference between the fluid within the boundary layer and free stream is small, so ${T^{\prime}}^{4}$ can be expressed as a linear combination of the temperature, we expand ${T^{\prime}}^{4}$ in Taylor's series about $T_{\infty}{ }^{\prime}$ and neglecting higher order terms, we get

$T^{\prime 4} \cong 4 T_{\infty}^{\prime 3} T^{\prime}-3 T_{\infty}^{\prime 4}$

Input (5) and (6) in (2)

$\frac{\partial T^{\prime}}{\partial t^{\prime}}=\frac{k}{\rho c_{p}} \frac{\partial^{2} T^{\prime}}{\partial y^{\prime 2}}-\frac{1}{\rho c_{p}} 16 a^{*} \sigma^{*} T_{\infty}^{\prime 3}\left(T^{\prime}-T_{\infty}^{\prime}\right)$

Where 
$y=\frac{y^{\prime}}{U_{0} t_{0}}, u=\frac{u^{\prime}}{U_{0}}, t=\frac{t^{\prime}}{t_{0}}, \theta=\frac{\left(T^{\prime}-T_{\infty}^{\prime} \infty\right)}{\left(T^{\prime} w^{-} T_{\infty}^{\prime}\right)}, C=\frac{\left(C^{\prime}-C_{\infty}^{\prime}\right)}{\left(C^{\prime} w^{-} C_{\infty}^{\prime}\right)}, G r=\frac{v g \beta^{\prime}{ }_{T}\left(T^{\prime}{ }_{w}-T_{\infty}^{\prime}\right)}{U_{0}^{3}}, \gamma=\frac{\mu_{B} \sqrt{2 \pi_{C}}}{P_{y}}$

$G m=\frac{v g \beta_{C}{ }_{C}\left(C^{\prime}{ }^{-}-C^{\prime}{ }_{\infty}\right)}{U_{0}^{3}}, M=\frac{\sigma B_{0}^{2} v}{\rho U_{0}^{2}}, P_{r}=\frac{\rho v C_{p}}{k}, R=\frac{16 a \sigma v^{2} T^{\prime}{ }_{\infty}{ }^{3}}{k U_{0}^{2}}, S c=\frac{v}{D_{M}}, R^{\prime}=\frac{v k_{2}^{\prime}}{U_{0}^{2}}, \tau=\frac{\tau}{\rho u^{2}}$

In equations (1-4) and equation (7) dropping out the " $"$ " notation (for simplicity) we get

$\frac{\partial u}{\partial t}=\left(1+\frac{1}{\gamma}\right) \frac{\partial^{2} u}{\partial y^{2}}-\left(M^{2}+\frac{1}{k}\right) u+G_{r} \theta+G_{m} C$

$\frac{\partial \theta}{\partial t}=\frac{1}{P r} \frac{\partial^{2} \theta}{\partial y^{2}}-\frac{R}{P r} \theta$

$\frac{\partial C}{\partial t}=\frac{1}{s_{c}} \frac{\partial^{2} C}{\partial y^{2}}-R^{\prime} C$

With initial and boundary condition

$u=\theta=C=0, \quad y \geq 0, t \leq 0$

$u=t^{2}, C=t, \theta=\left\{\begin{array}{rr}t, & 0<t \leq 1 \\ 1 & t>1\end{array}\right.$ at $y=0, t>0$

$u \rightarrow 0, \theta \rightarrow 0, C \rightarrow 0$ at $y \rightarrow \infty, t>0$

\section{Solution of the problem}

$\theta(y, t)=\theta_{1}(y, t)-\theta_{1}(y, t) H(t-1)$

$C(y, t)=\frac{1}{2}\left[\left(t-\frac{y \sqrt{S c}}{2 \sqrt{R^{\prime}}}\right) e^{-y \sqrt{R^{\prime}}} \operatorname{erfc}\left(\frac{y \sqrt{S c}}{2 \sqrt{t}}-\sqrt{R^{\prime} t}\right)+\left(t+\frac{y \sqrt{S c}}{2 \sqrt{R^{\prime}}}\right) e^{y \sqrt{R^{\prime}}} \operatorname{erfc}\left(\frac{y \sqrt{S c}}{2 \sqrt{t}}+\sqrt{\frac{R^{\prime}}{\operatorname{Pr}}} t\right)\right]$

$u(y, t)=f_{1}(y, t)+f_{4}(y, t)+f_{9}(y, t)$

\subsection{Solutions for Plate with Constant Temperature}

In this case, the initial and boundary conditions are the same excluding Eq. (11) that becomes $\theta=$ 1 at $y=0, t \geq 0$.We find the isothermal temperature $\theta(y, t)$ using Laplace transform.

$$
\begin{aligned}
& \theta(y, t)=\frac{1}{2}\left[e^{-y \sqrt{R}} \operatorname{erfc}\left(\frac{y \sqrt{\operatorname{Pr}}}{2 \sqrt{t}}-\sqrt{\frac{R}{P r}} t\right)+e^{-y \sqrt{R}} \operatorname{erfc}\left(\frac{y \sqrt{P r}}{2 \sqrt{t}}+\sqrt{\frac{R}{P r} t}\right)\right] \\
& u=f_{1}(y, t)+\frac{G r}{d} f_{14}(y, t)+\frac{G m}{g} f_{10}(y, t)
\end{aligned}
$$

Where $a=1+\frac{1}{\gamma}, b=M^{2}+\frac{1}{k}, a R-b=c, a P r-1=d, \frac{c}{d}=e, \quad s_{c} R^{\prime}-b=f$,

$\mathrm{aSc}-1=\mathrm{g}, \frac{\mathrm{f}}{\mathrm{g}}=\mathrm{h}$,

$\theta_{1}(y, t)=\frac{1}{2}\left[\left(t-\frac{y \sqrt{\operatorname{Pr}}}{2 \sqrt{\frac{R}{\operatorname{Pr}}}}\right) e^{-y \sqrt{\mathrm{R}}} \operatorname{erfc}\left(\frac{\mathrm{y} \sqrt{\operatorname{Pr}}}{2 \sqrt{\mathrm{t}}}-\sqrt{\frac{\mathrm{R}}{\operatorname{Pr}} \mathrm{t}}\right)+\left(\mathrm{t}+\frac{\mathrm{y} \sqrt{\mathrm{Pr}}}{2 \sqrt{\frac{\mathrm{R}}{\operatorname{Pr}}}}\right) \mathrm{e}^{\mathrm{y} \sqrt{\mathrm{R}}} \operatorname{erfc}\left(\frac{\mathrm{y} \sqrt{\operatorname{Pr}}}{2 \sqrt{\mathrm{t}}}+\sqrt{\frac{\mathrm{R}}{\operatorname{Pr}} \mathrm{t}}\right)\right]$

$f_{1}(y, t)=\int_{0}^{t} f_{2}(t-s) f_{3}(y, s) d s, f_{2}(y, t)=2 t$

$f_{3}(y, t)=\frac{1}{2}\left[e^{-y \sqrt{\frac{b}{a}}} \operatorname{erfc}\left(\frac{y \frac{1}{\sqrt{a}}}{2 \sqrt{t}}-\sqrt{b t}\right)+e^{-y \sqrt{\frac{b}{a}}} \operatorname{erfc}\left(\frac{y \frac{1}{\sqrt{a}}}{2 \sqrt{t}}+\sqrt{b t}\right)\right]$

$f_{4}(y, t)=\frac{G_{r}}{d}\left(f_{4}(y, t) H(t)-f_{4}(y, t) H(t-1)\right), \mathrm{f}_{5}(\mathrm{y}, \mathrm{t})=\int_{0}^{\mathrm{t}} \mathrm{f}_{6}(\mathrm{t}-\mathrm{s}) \mathrm{f}_{7}(\mathrm{y}, \mathrm{s}) \mathrm{ds}$

$f_{6}(y, t)=\frac{1}{e}-\frac{1}{e} e^{-e t}, f_{7}(y, t)=f_{3}(y, t)-f_{8}(y, t)$ 


$$
\begin{aligned}
& f_{8}(y, t)=\frac{1}{2}\left[e^{-y \sqrt{R}} \operatorname{erfc}\left(\frac{y \sqrt{P r}}{2 \sqrt{t}}-\sqrt{\frac{R}{P r}} t\right)+e^{-y \sqrt{R}} \operatorname{erfc}\left(\frac{y \sqrt{P r}}{2 \sqrt{t}}+\sqrt{\frac{R}{P r}} t\right)\right] \\
& f_{9}(y, t)=\frac{G_{m}}{g} f_{10}(y, t), f_{10}(y, t)=\int_{0}^{t} f_{11}(t-s) f_{12}(y, s) d s \\
& f_{11}(y, t)=\frac{1}{h}-\frac{1}{h} e^{-h t}, f_{12}(y, t)=f_{3}(y, t)-f_{13}(y, t) \\
& f_{13}(y, t)=\frac{1}{2}\left[e^{-y \sqrt{R^{\prime} S c}} \operatorname{erfc}\left(\frac{y \sqrt{S c}}{2 \sqrt{t}}-\sqrt{R^{\prime} t}\right)+e^{-y \sqrt{R^{\prime} S c}} \operatorname{erfc}\left(\frac{y \sqrt{S c}}{2 \sqrt{t}}+\sqrt{R^{\prime} t}\right)\right] \\
& f_{14}(y, t)=\int_{0}^{t} f_{15}(t-s) f_{7}(y, s) d s, F_{15}(y, t)=e^{-c t}
\end{aligned}
$$

\section{Results and discussion}

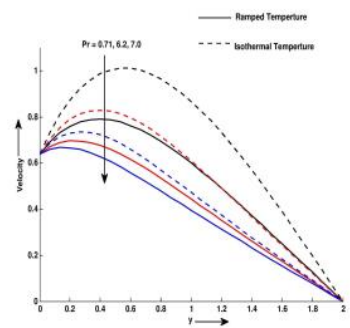

Figure 2:

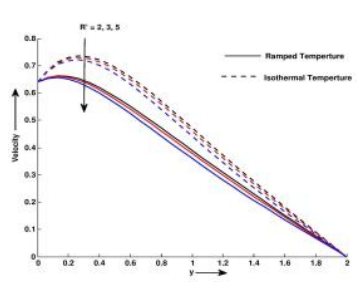

Figure 6:

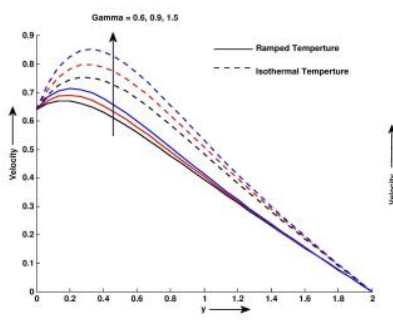

Figure 3:

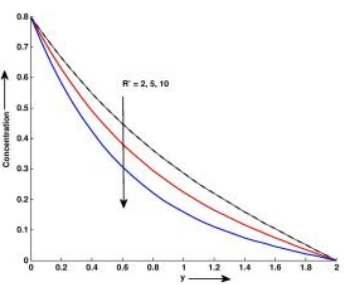

Figure 7:

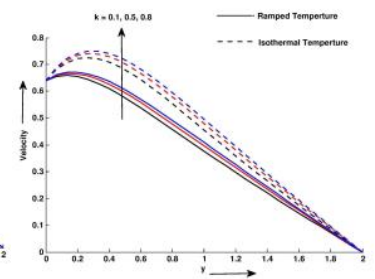

Figure 4:

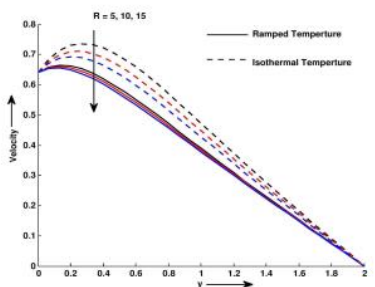

Figure 8:

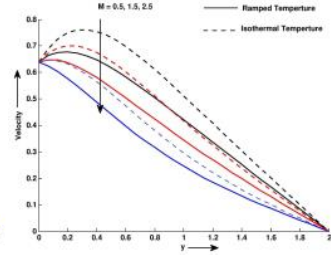

Figure 5:

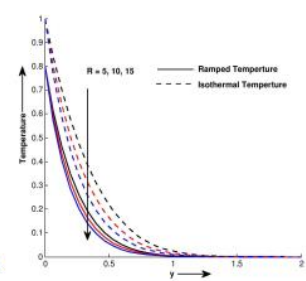

Figure 9:

We have presented the non-dimensional fluid velocity, fluid temperature and concentration for several values of different physical parameter. Figure 2 exhibits the velocity profiles for different values of Prandtl number Pr, when the other parameters are fixed. It is observed that velocity of the fluid decreases with increasing Prandtl number. The influence of Casson fluid parameter on velocity profiles is shown in Figure 3. It is found that velocity increases with increasing value of $\gamma$. It is important to note that an increase in Casson parameter makes the velocity boundary layer thickness shorter. Decrease in Casson parameter results in increase in the plasticity of the fluid which causes the increment in velocity boundary layer thickness. In Figure 4, the profiles of velocity have been plotted for various values of permeability parameter $\mathrm{K}$, keeping other parameters fixed. It is observed that for large values of $\mathrm{K}$, velocity and boundary layer thickness increase. This explains the physical situation that as $\mathrm{K}$ increases, the resistance of the porous medium is lowered which increase the momentum development of the flow regime, ultimately enhances the velocity field. Figure 5 displays the effect of magnetic parameter $\mathrm{M}$ on the velocity profiles. It is observed that the amplitude of the velocity as well as the boundary layer thickness decreases when $\mathrm{M}$ is increased. Physically, it may also be expected due to the fact that the application of a transverse magnetic field results in a resistive type force (called Lorentz force) similar to the drag force, and upon increasing the values of $\mathrm{M}$, the drag force increases which leads to the deceleration of the flow. Chemical reaction has a retarding influence on fluid flow velocity and concentration profile for both thermal cases as shown in Figure 6 and Figure 7. This shows that the destructive reaction $\mathrm{Kr}>0$ leads to fall in the concentration field, which in turn weakens the buoyancy effects due to concentration gradients. Consequently, the flow field is retarded. 
This occurrence has a superior agreement with the physical realities. Thermal radiation parameter has a retarding influence on fluid flow velocity and temperature profile for both thermal cases as shown in Figure 8-9. It is notice that thermal radiation parameter reduces thermal buoyancy force, minimizing the thickness of the thermal boundary layer. Therefor velocity and temperature profiles increase with radiation parameter R. Physically, when the amount of heat generated through thermal radiation parameter increases, the bond holding the components of the fluid particles is easily broken and the fluid velocity will increase.

\section{Conclusion}

The most important concluding remarks can be summarized as follows:

- Velocity and temperature profiles in case of ramped wall temperature are less than that isothermal temperature but effect of all parameters are similar in both thermal cases.

- In both thermal cases, Velocity decreases with increase in Prandtl number Pr, magnetic parameter $\mathrm{M}$, radiation parameter $\mathrm{R}$ and chemical reaction parameter $R^{\prime}$.

- In both thermal cases, Velocity increases with increasing values of permeability of porous medium $\mathrm{k}$.

- Thermal radiation parameter tends to reduced heat transfer process.

- Chemical reaction parameter tends to reduced mass transfer process.

\section{References}

[1] N. Casson, A flow equation for the pigment oil suspensions of the printing ink type, in: Rheology of Disperse Systems, Pergamon, NewYork, 1959, pp. 84-102.

[2] R.K. Dash, K.N. Mehta, G. Jayaraman, Casson fluid flow in a pipe filled with a homogeneous porous medium, Int. J. Eng. Sci. 34 (1996) 1145-1156

[3] H. Kataria and H. Patel. Heat and Mass Transfer in MHD Second Grade Fluid Flow with Ramped Wall Temperature through Porous Medium. Mathematics Today, 32, (2016) 67-83.

[4] S. Nadeem, Rizwan Ul Haq, C. Lee. MHD flow of a Casson fluid over an exponentially shrinking sheet. Scientia Iranica B (2012) 19 (6), 1550-1553

[5] K,Das. Effect of chemical reaction and thermal radiation on heat and mass transfer flow of MHD micropolar fluid in a rotating frame of reference. International Journal of Heat and Mass Transfer, 54, (2011), 3505-3513

[6] H. R. Kataria, A. S. Mittal, Mathematical model for velocity and temperature of gravitydriven convective optically thick nanofluid flow past an oscillating vertical plate in presence of magnetic field and radiation. Journal of Nigerian Mathematical Society, 34 (2015) 303-317 
[7] H. R. Kataria, A. S. Mittal, Velocity, mass and temperature analysis of gravity-driven convection nanofluid flow past an oscillating vertical plate in presence of magnetic field in a porous medium, Applied Thermal Engineering, 110 (2017) 864-874

[8] H. R. Kataria, H. R. Patel, R. K. Singh, Effect of magnetic field on unsteady natural convective flow of a micropolar fluid between two vertical walls, Ain Shams Engineering Journal, DOI:10.1016/j.asej.2015.08.013.

[9] I. Muhaimin, R. Kandasamy, A. B. Khamis, R. Roslan. Effect of thermophoresis particle deposition and chemical reaction on unsteady MHD mixed convective flow over a porous wedge in the presence of temperature-dependent viscosity. Nuclear Engineering and Design, Volume 261, August 2013, Pages 95-106

[10] R. Muthucumaraswamy, E. Geetha. Effects of parabolic motion on an isothermal vertical plate with constant mass flux. Ain Shams Engineering Journal, 5 (2014) 1317-1323

[11] H. R. Kataria, H. R. Patel, Radiation and chemical reaction effects on MHD Casson fluid flow past an oscillating vertical plate embedded in porous medium, Alexandria Engineering Journal , 55 (2016) 583-595

[12] H. R. Kataria, H. R. Patel, soret and heat generation effects on MHD casson fluid flow past an oscillating vertical plate embedded through porous medium, Alexandria Engineering Journal, 55 (2016) 2125-2137

[13] H. R. Kataria, H. R. Patel, Effect of thermo-diffusion and parabolic motion on MHD Second grade fluid flow with ramped wall temperature and ramped surface concentration, Alexandria Engineering Journal, 10.1016/j.aej.2016.11.014

[14] S. Rosseland, (1931), Astrophysik und atom-theoretische Grundlagen. Berlin: SpringerVerlag.

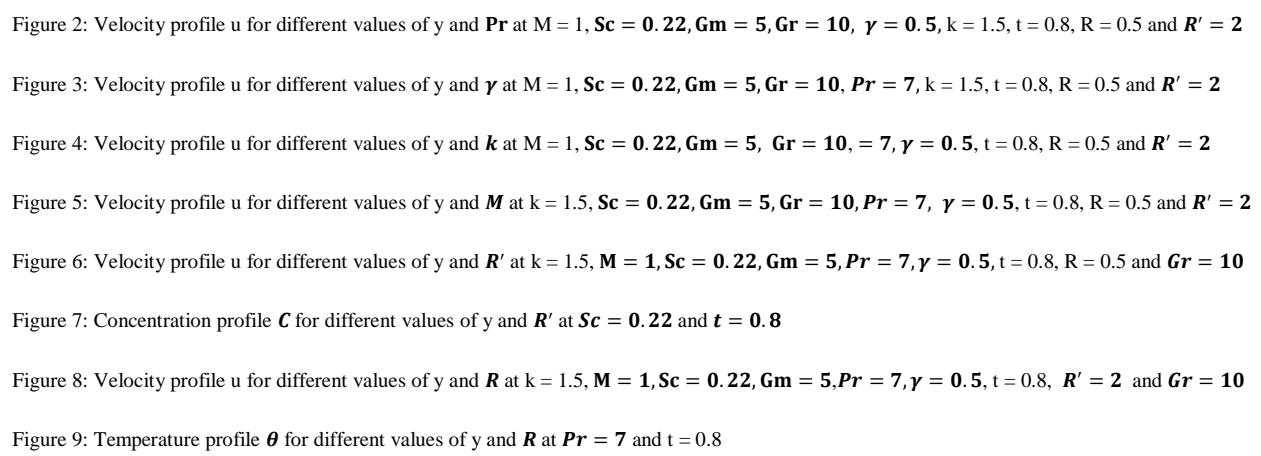

